\title{
Automatic SystemC Code Generation from UML Models at Early Stages of Systems on Chip Design
}

\author{
Fateh Boutekkouk \\ Larbi ben M'hedi University \\ BP 358, Oum El Bouaghi, 04000 \\ Algeria
}

\begin{abstract}
In this paper, we present our approach for automatic SystemC code generation from UML models at early stages of Systems On Chip (SOC) design. A particularity of our proposed approach is the fact that SystemC code generation process is performed through two levels of abstraction. In the first level, we use UML hierarchic sequence diagrams to generate a SystemC code that targets algorithmic space exploration and simulation. In the second level of abstraction, messages that occur in sequence diagrams are implemented using UML activity diagrams whose actions are expressed in the $\mathrm{C}++$ Action Language (AL) included in the Rhapsody environment from which a full SystemC code is generated for both simulation and synthesis.
\end{abstract}

\section{General Terms}

Systems On Chip, System Modeling

\section{Keywords}

UML, SystemC, Sequence diagrams, Activity diagrams, Action Language, Simulation.

\section{INTRODUCTION}

System On Chip (SOC) [1] can be defined as a complex integrated circuit that integrates the major functional elements of a complete end-product into a single chip or chipset.

The ever complexity of SOC design has pushed researchers in the field to raise the level of abstraction and exploit recent Software Engineering technologies such as object technology and in particular the Unified Modeling Language (UML) [2, 3].

SOC designers are now confronted with the challenge of how to bridge the gap between software standard modeling language such as UML and the well practiced SOC System Level Languages (SLL) like SystemC [4, 5, 6]. Since UML was initially introduced in the software domain, most commercial tools generate software code such as $\mathrm{C}, \mathrm{C}++$, and Java from UML models. However, there is a lack of tools that can synthesize UML models into SLL descriptions. Our objective is to raise the level from which SystemC descriptions can be generated to perform quick simulations and synthesis eventually. Thus a refinement directed approach seems inevitable to bridge the gap smoothly between UML models and SystemC descriptions. To address this problem, we have proposed a flow that permits automatic SystemC code generation from UML models at two levels of abstraction. The first level corresponds to SystemC code generation from UML sequence diagrams without implementing messages. Thus the generated code at this stage is oriented to algorithmic space exploration and simulation since the obtained code consists only of processes input/output ports, processes sensitivity lists, dependencies between processes, and signals. The second level of abstraction is a refinement of the first level where messages are implemented using UML activity diagrams whose state actions are expressed in the Action Language included in the Rhapsody environment [7]. At this stage, the generated code is dedicated to both simulation and synthesis. The rest of this paper is organized as follows: section two is dedicated to related works concerning the synthesis of UML models to SystemC code. Section three puts the light on UML. Section four gives an overview of the SystemC language. Our proposed flow with an illustrative example is discussed in section five. Section six is about implementation and a case study before concluding.

\section{RELATED WORK}

In this section, we try to present brievely some pertinent works targeting the generation of SystemC code from UML models.

SystemC code generation from UML was first investigated in [8], who presented several benefits when combining UML/SysML and SystemC, like a common and structured environment for system documentation/specification. The approaches in [9] and [10] cope with direct code generation by taking the UML model as an XMI (XML Metadata Interchange) file for translation to SystemC.

In [11], the authors presented a UML/SystemC profile for SystemC code generation from UML structural and Statecharts diagrams. In [12], the authors proposed a UML/MDA approach called MoPCoM methodology that permits automatic SystemC and VHDL code generation from UML models and MARTE profile by means of MDA techniques. Input models are focused on UML class, component, and Statecharts diagrams.

In [13], an approach to bridge the gap between UML and SystemC is presented. The proposed framework permits the integration of a customized SysML [16] (SysML) is an extension of UML for systems engineering entry with the code generation for HW/SW cosimulation and high level FPGA synthesis. Input models are focused on classes and blocks diagrams.

As opposite to these works, our approach tries to generate SystemC code automatically at early stages of SOC design (requirement analysis) from UML sequence diagrams in a first step then from UML activity diagrams in a second step.

\section{THE UNIFIED MODELING LANGUAGE}

UML [2] is a graphical object-oriented modeling language, initially, was used in software systems. However, and according to authors, UML can be tailored to SOC domain [14]. 
In our case, we have chosen the Rhapsody environment [7] for UML modeling, functional simulation, and automatic SystemC code generation.

\section{SYSTEMC}

SystemC [4] is an extension of $\mathrm{C}++$ language for SOC modeling and simulation. It represents a standard for SOC system level modeling. Various versions of the language have appeared but we consider SystemC2.0.

SystemC structural designs are focused on modules.

A module contains ports, interfaces, channels, processes, and eventually other modules. In SystemC, concurrent behaviors are modeled using processes. A process has a sensitivity list that includes the set of signals to which it is sensitive. This list can be either static (pre-specified before simulation starts) or dynamic.

SystemC processes execute concurrently and may suspend on wait() statements. Such processes requiring their own independent execution stack are called "SC_THREADs". When the only signal triggering a process is the clock signal ' $c l k$ ' we obtain what we call "SC_CTHREAD" (clocked thread process). Certain processes do not actually require an independent execution stack and cannot suspended on wait() statement. Such processes are termed "SC_METHODs". SC_METHOD processes execute in zero simulation time and returns control back to the simulation kernel.

The following code [6] presents a SystemC module named display with an input port din, and a SC_METHOD called print_data which is sensible to din. For each SystemC module there are two files: $h$ for ports, functions, variables, and processes declaration and.$c c$ for process and functions implementation. systemc. $h$ designates the SystemC library file.

// display.h
\#include "systemc.h"
\#include "packet.h"
SC_MODULE(display) \{
sc_in<long>din; // input port
void print_data();
//Constructor
SC_CTOR(display) \{
SC_METHOD(print_data); //Method process to print data
sensitive <<din;
H\};
// display.cc
\#include "display.h"
void display::print_data() \{
cout <<"Display:Data Value Received, Data = "<< din $<<$
" $n$ ";

\section{OUR FLOW}

As illustrated in figure 1, our proposed flow starts by capturing system requirements as a set of related uses cases and actors. At this stage, we use UML use cases diagrams with 'include' and 'extend' relations. Figure 2 gives an example of modelling with use cases diagram. In this example, we have one actor and two use cases named usecase_O and usecase_1. usecase_O is related to usecase_l by the 'include' relation. Each use case diagram is then refined to a set of interacting objects exhibiting a possible scenario. At this stage, we use UML sequence diagrams. The 'include' relation is modelled as an unconditional call of the use case child while the 'extend' relation is an optional call subject to some condition. Figure 3 shows a possible implementation of use cases using hierarchic sequence diagrams. In this example, we model usecase_0 as the parent use case using sequence diagram with three interacting objects (class's instances) class_0, class_1, and class_2 and an external object that represents the environment $(E n v)$. usecase_l is modelled as a child sequence diagram invoking by a call from the environment. In order to model the 'extend' relation, we add a conditional call invoking the child sequence diagram (usecase_2 in figure 4). From UML sequence diagrams, a SystemC code is generated automatically using the VB API which is integrated in the Rhapsody environment. This API offers the necessary functions and commands that permit the parsing of UML diagrams and then the extraction of information needed for SystemC code generation as text files. The generated code in this step will be used for algorithmic space exploration and simulation eventually. We have used three techniques for SystemC code generation process. In the first technique, each message is considered as a SystemC SC_METHOD. In the second technique, each end-toend scenario is considered as a SystemC SC_THREAD. In the third technique, each object is considered as a SystemC SC_THREAD. Dashed lines in figure 1 enable the designer to modify his/her design according to simulation results.

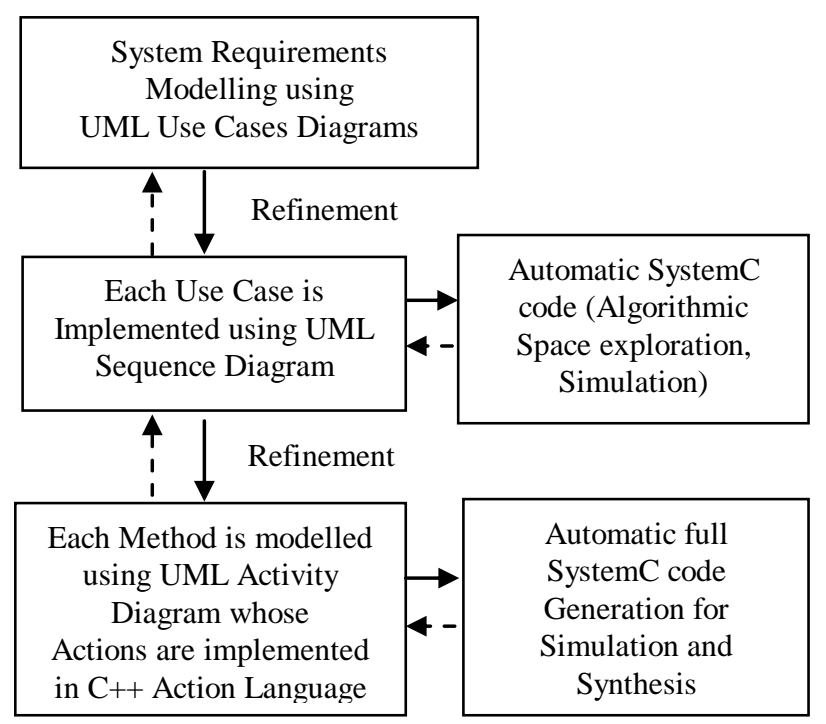


Figure 1. Our proposed flow

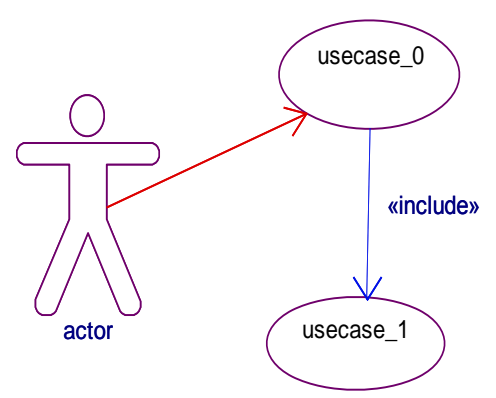

Figure 2. Example of UML use cases diagram

\subsection{Illustrative example}

In order to motivate our proposed approach, we apply the code generation process on an example whose use case diagram is illustrated in figure 2. In this example, we assume that we have an actor and two use cases named usecase_0 and usecase_l that are related by an 'include' relation. Both usecase_O and usecase_ 1 are implemented using UML sequence diagrams as showed in figure 5 . In the following sections, we try to explain the three techniques for SystemC code generation from UML sequence diagrams.

\subsection{First technique}

In this technique, each message is mapped to a SystemC SC_METHOD. Methods arguments are transformed to input ports while returned values are mapped to output ports. To each call to a message, we add a Boolean input port that corresponds to the event to which process is sensible and a Boolean output port that corresponds to control return. From figure 5, we observe that message_2 is used in both usecase_0 and usecase_1. Such a common message will be mapped to a SC_METHOD process in a separate module. It is obvious, that this technique may lead to a very big number of fine grained processes which is not acceptable in complex designs. But it serves as a first solution for algorithmic space exploration. Table 1 shows the correspondence between UML and SystemC concepts.

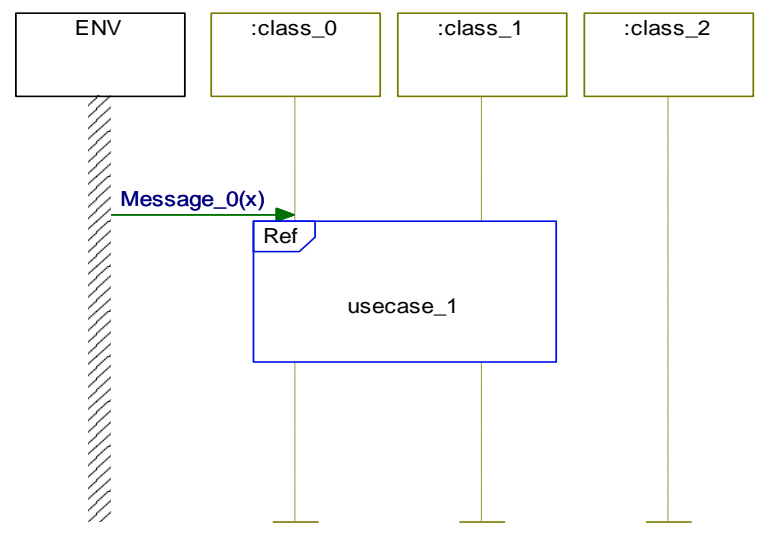

Figure 3. Possible implementation of 'include' relation

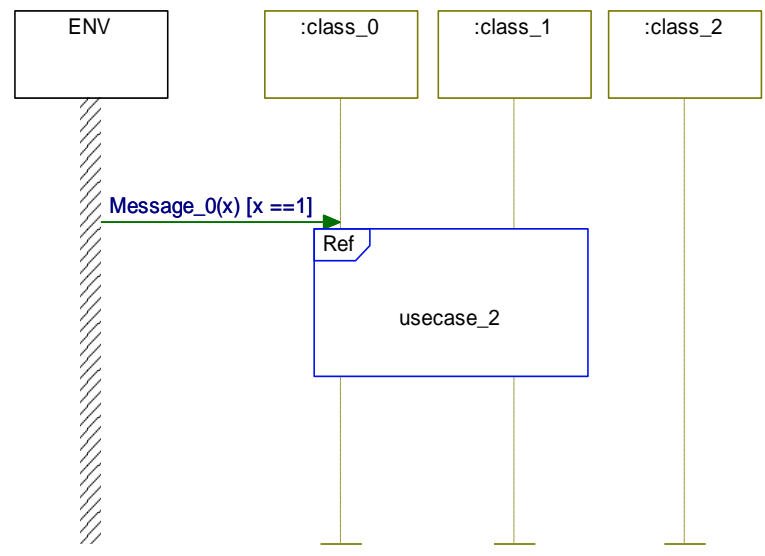

Figure 4. Possible implementation of 'extend' relation

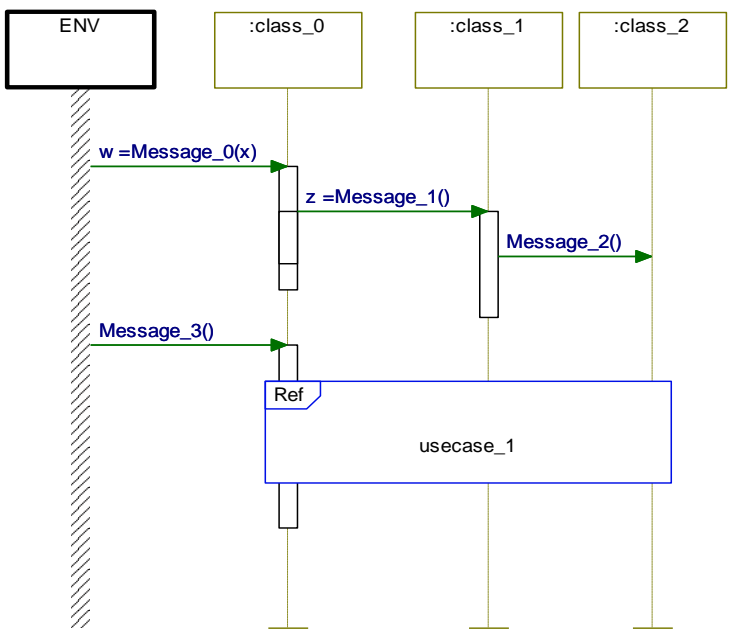

(a)

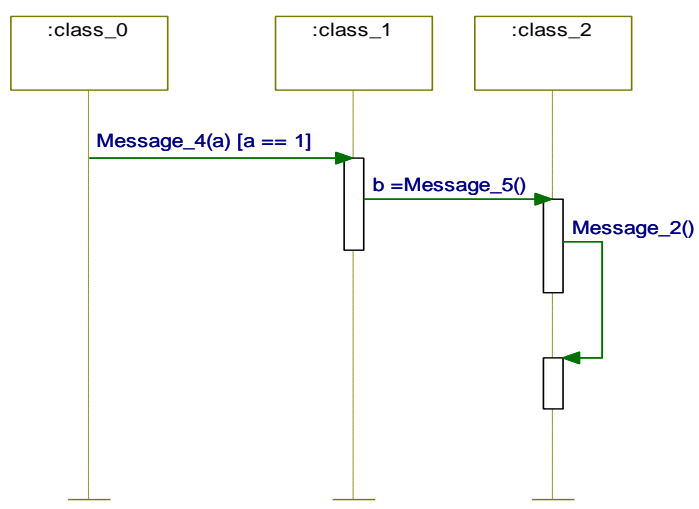

(b)

Figure 5. Example of hierarchic sequence diagrams

(a) Parent sequence diagram (usecase_0); (b) Child sequence diagram (usecase_1) 
Table 1. Correspondence between UML and SystemC for the first technique

\begin{tabular}{|c|c|}
\hline UML concept & SystemC concept \\
\hline message & SC_METHOD \\
\hline Common message & $\begin{array}{c}\text { SC_METHOD in a } \\
\text { separate module }\end{array}$ \\
\hline argument & sc_in<type $>$ port \\
\hline Return value & sc_out $<$ type $>$ port \\
\hline call & sc_inout $<$ bool $>$ \\
\hline Control return & sc_out $<$ bool $>$ \\
\hline $\begin{array}{c}\text { Child sequence } \\
\text { diagram }\end{array}$ & Sub module \\
\hline $\begin{array}{c}\text { Top level model } \\
\text { So_main( })\end{array}$ \\
\hline
\end{tabular}

Assume that we have a message with two integer arguments ( $a$ and $b$ ) and an integer return value $x: x=$ message $(a, b)$.

The corresponding SystemC code for this message is as follows:

// module1.h

\# include "systemc.h"

SC_MODULE(module1)!

$s c \_$in $\langle$int $>a$;

$s c \_$in $\langle$int $>b$;

$s c \_$out $<$int $>x$;

sc_inout $<$ bool $>$ cal;

sc_out $<$ bool $>$ ret;

void message();

SC_CTOR(module1) \{

SC_METHOD(message);

sensitive $<$ cal; \}\};

// module1.cc

\#include "module1.h"

void module $1:$ message() \{

int arg1, arg2, result;

while cal $==0$;

cal $=0 ; \quad / /$ cal = false;

$\arg 1=a$;

$\arg 2=b$

// message body

$x=$ result;

ret $=1 ;\} \quad$ // ret $=$ true;

SC_METHOD message is sensitive to the signal cal.

arg 1 and $\arg 2$ are two variables used to stock the two arguments coming from the two ports $a$ and $b$.

result is a variable used to stock the returned value in the port $x$. We use the Boolean ports cal and ret to specify the message invoking and the return of the control to the caller respectively. The meaning of this SystemC code is as follows:

The process message will be blocked until the occurrence of the signal $\mathrm{cal}(\mathrm{cal}=1)$. After that, the process resumes its execution; sets cal to false; stocks the arguments coming from input ports $a$ and $b$ into variables $\arg 1$ and $\arg 2$; performs some computation; stocks the result of computation into output port $x$; sets the signal ret to true. Similarly, The SystemC code for the caller is as follows:

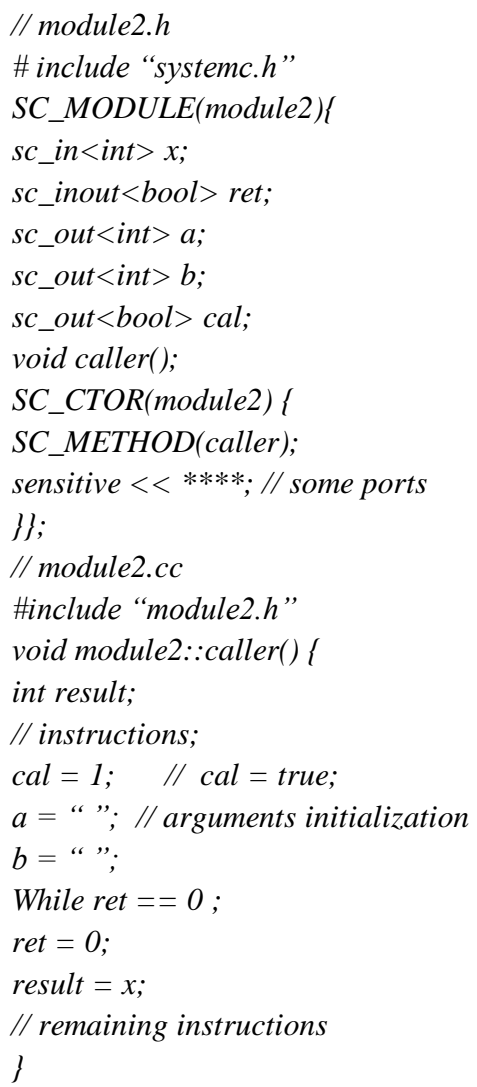

Note that SC_METHOD processes message and caller are put in two distinct modules: modulel and module 2 respectively. However, if we put them into one module, all ports become sc_inout. By applying this technique on our example, we obtain six (6) SC_METHOD processes that are: Message_0, Message_1, Message_2, Message_3, Message_4, and Message_5. Assume that all messages arguments and return values are integers. cal0, call, cal2, cal3, cal4, and cal5 designate Boolean ports for message_0, message_1, message_2, message_3 message_4, and message_5 calls respectively. arg0 and arg4 designate ports for message_O and message_4 arguments respectively. val0, vall, and val5 designate ports for message_0, message_1, and message_5 returned values respectively. ret 0 , ret 1 , ret 2 , ret3, ret4, and ret5 designate Boolean ports for messages controls return.

Since message_2 is a common message, we put it in a separate module called mess 2 . Here, we have two modules: usecase 0 including SC_METHODS message_0, message_1, and message_3, and usecaselincluding message_4, and message_5.

The corresponding SystemC code looks like:

// mess $2 . h$

\# include "systemc.h" 
SC_MODULE(mess2)

$s c \_$inout $<$bool $>$cal2;

$s c \_o u t<b o o l>r e t 2$;

void message_2();

SC_CTOR(mess 2$)$ i

$S C \_M E T H O D\left(m e s s a g e \_2\right)$;

sensitive $<$ cal2;

\});

// mess2.cc

\#include "mess 2.h"

void mess $2:: m e s s a g e \_2()\{$

while cal2 $==0$;

cal2 $=0$;

// message body;

ret $2=1 ;\}$

// usecase1.h

\# include "systemc.h"

SC_MODULE(usecase1)\{

$s c \_$in $\langle$int > arg4; sc_inout<int >val5; sc_out <bool>cal2;

$s c \_$inout $<$bool $>$ret 2; $s c \_$inout $<$bool $>$cal4;

$s c \_$inout $<$bool $>$cal5; $s c \_i n o u t<b o o l>r e t 5$; $s c \_o u t<b o o l>r e t 4$;

void message_4(); void message_5();

SC_METHOD(message_4);

sensitive $<$ cal4;

SC_METHOD(message_5);

sensitive $<$ cal5;

)\};

// usecase1.cc

void usecasel::message_4() (

int var, result;

while cal4 $==0$;

cal4 $=0$;

var = arg4;

// instructions

cal5 = 1 ;

while ret $5==0$;

ret5 $=0$;

result $=$ val5;

// remaining instructions

ret4 = 1 ;

\}

void usecase1::message_5() \{

// code

\}

// usecase0.h

\# include "systemc.h"

SC_MODULE(usecase0)\{

$s c \_$in $\left\langle i n t>a r g 0 ; s c \_i n o u t<i n t>a r g 4\right.$; sc_out $\langle$ int $>v a l 0$;

$s c \_i n o u t\langle i n t\rangle$ vall; sc_inout<bool> calo; $s c \_i n o u t\langle b o o l\rangle$ call;

$s c \_o u t<b o o l>c a l 2$; sc_inout $\langle$ bool $>$ cal3; sc_out $\langle$ bool $>$ cal4;

$s c \_o u t<b o o l>r e t 0$; $s c_{-}$inout $<$bool $>$ret 1 ; $s c_{-}$inout $<$bool $>r e t 2$; $s c \_o u t<b o o l>r e t 3 ; s c \_i n o u t<b o o l>r e t 4$;

void message_O(); void message_1(); void message_3();

SC_CTOR(usecaseO) i

$S C \_M E T H O D($ message_O);

sensitive $<<$ calO;

SC_METHOD(message_1);

sensitive $<$ call,

SC_METHOD(message_3);

sensitive $<<$ cal3;

\});

// usecase0.cc

\#include "usecase0.h"

void usecase0::message_O() \{

// code

\};

void usecasel::message_1() \{

// code

;

void usecase $1:$ message_3() \{

int var;

while cal3 $==0$;

cal3 $=0$;

// instructions

$\arg 4=$ var;

if $\arg 4=1$ l

cal4 = 1;

while ret $4==0$;

ret4 $=0$;

\}

// remaining instructions

ret3 $=1$

\};

// main.cc

\#include "mess 2.h"

\#include "usecase1.h"

\#include "usecase0.h"

int sc_main(int argc, char* argv[]) \{

sc_signal<int>ARG0, ARG4,VAL0,VAL1;

sc_signal<bool>CAL0,CAL1,CAL2,CAL3,CAL4,CAL5 ;

sc_signal<bool> RETO, RET1, RET2, RET3, RET4, RET5 ;

mess2 ms2("mess2"); ms2.cal2(CAL2); ms2.ret2(RET2);

usecasel ucl("usecasel");

ucl.arg4(ARG4); ucl.val5(VAL5);uc1.cal2(CAL2);

ucl.cal4(CAL4);uc1.cal5(CAL5);uc1.ret2(RET2);uc1.ret4(RET4)

;uc1.ret5(RET5);

usecase0 uc0(“usecase0");

uc0.argO(ARG0); uc0.arg4(ARG4);uc0.valO(VALO);

uc0.val1(VAL1);uc0.calO(CALO);uc0.call(CAL1);

uc0.cal2(CAL2);uc0.cal3(CAL3);uc0.cal4(CAL4);

uc0.retO(RETO); uc0.ret1(RET1);uc0.ret2(RET2);

uc0.ret3(RET3); uc0.ret4(RET4);

return $(0) ;\}$ 


\subsection{Second technique}

In this technique, we consider each end-to-end scenario as a SystemC SC_THREAD. An end-to-end scenario is a sequence of methods that are invoked by an external call from the environment. Table 2 shows the correspondence between UML and SystemC concepts. All internal methods are implemented as SystemC functions.

Table 2. Correspondence between UML and SystemC for the second technique

\begin{tabular}{|c|c|}
\hline UML concept & SystemC concept \\
\hline End-to-end scenario & SC_THREAD \\
\hline Internal message & C++ function \\
\hline External call & port \\
\hline Top level model & sc_main( $)$ \\
\hline
\end{tabular}

By applying this technique on the above example, we obtain two SystemC SC_THREADS: process 1 including the sequence of messages: message_0, message_1, and message_2 and process 2 including message_3, message_4, message_5, and message_2. process 1 is sensitive to cal0 and process 2 to cal3.

The corresponding SystemC code is as follows:

// system.h

\# include "systemc.h"

SC_MODULE(system)\{

$s c \_$in $\left\langle\right.$int $>$arg0; $s c \_i n o u t<b o o l>c a l 0$; $s c \_i n o u t<b o o l>c a l 3$;

$s c \_o u t<b o o l>r e t 0$; $s c \_o u t<b o o l>r e t 3$; $s c \_o u t<b o o l>v a l 0$;

int message_O(int); int message_1(void) ; void message_2(void);

void message_3(void); void message_4(int); int

message_5(void);

void process1(); void process $2($ );

SC_CTOR(system) \{

SC_THREAD(process 1);

sensitive $<$ calo;

SC_THREAD(process 2 );

sensitive $<$ cal3;

)\};

// system.cc

void message_2(void)\}

// message_2 body\}

int message_1(void)

// instructions

message_2() ; // call to message_2

// remainig instructions

int message_O(int) \{

int result;

// instructions

Result $=$ message_1();

// remaining instructions

return\} int message_5(void) \{

// instructions

message_2();

// remaining instructions

Return\}

void message_4(int) \{

int result;

// instructions

Result $=$ message_5();

// remaining instructions

void message_3(void) \{

int arg ;

// instructions

if arg == 1 message_4(arg) ;

// remaining instructions\}

void system::process 1() \{

wait();

calo $=0$

$\arg =\arg 0$;

valO $=$ message_O(arg $)$;

ret0 $=1 ;\}$

void system::process 2() (

wait();

cal3 $=0$;

message_3();

ret3 $=1 ;\}$

// main.cc

\#include "system.h"

int sc_main(int argc, char* argv[]) \{

sc_signal<bool>CALO,CAL3, RETO, RET3;

sc_signal<int $>A R G O, V A L O$;

system sys("system");

sys.argO(ARGO); sys.calO(CALO); sys.cal3(CAL3);

sys.retO(RETO); sys.ret3(RET3); sys.valO(VALO);

$\operatorname{return}(0) ;$;

\subsection{Third technique}

In this technique, each UML object is considered as a SC_THREAD. For each input /output message call, we create input/output ports (we add more ports for arguments and returned values). Table 3 shows the correspondence between UML and SystemC concepts. By applying this technique on the above example, we obtain four processes (4): Env, class_O, class_1, and class_2.

Table 3. Correspondence between UML and SystemC for the third technique

\begin{tabular}{|c|c|}
\hline UML concept & SystemC concept \\
\hline Object & SC_THREAD \\
\hline Input message call & Input ports \\
\hline
\end{tabular}




\begin{tabular}{|c|c|}
\hline output message call & Output ports \\
\hline Top level model & $s c \_$main() \\
\hline
\end{tabular}

For the sake of space, we give only the SystemC code for Env and class_O.

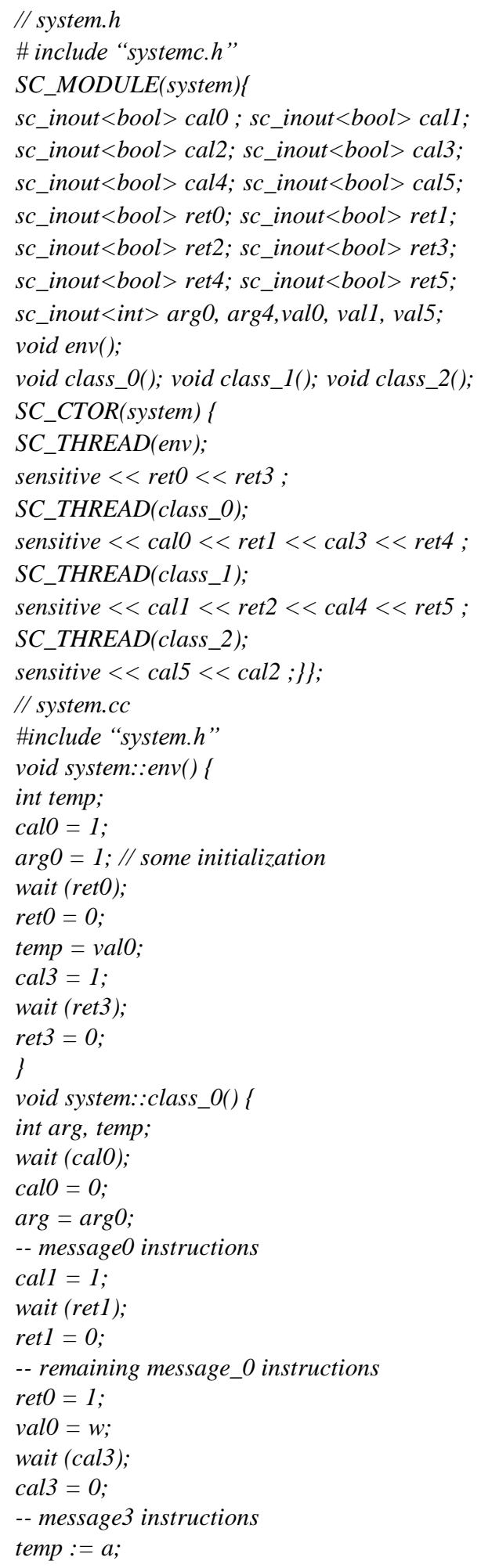

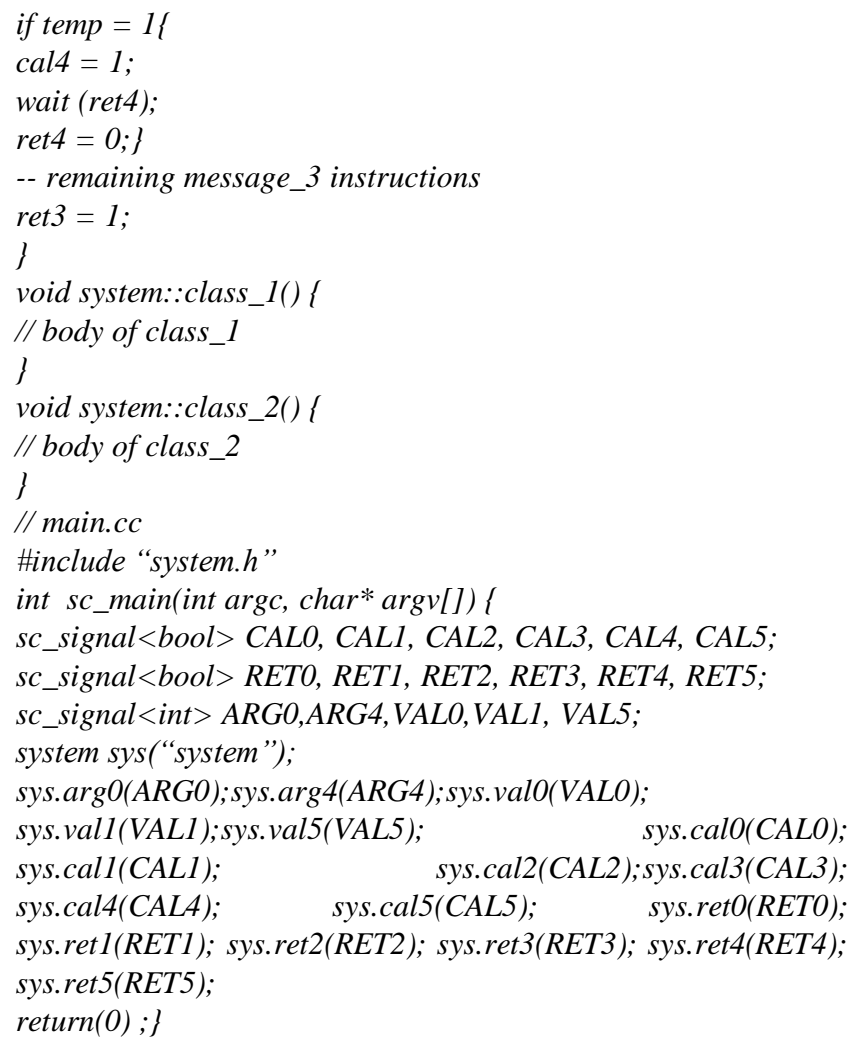

\subsection{Modeling with UML activity diagrams}

In our proposed flow (see figure 1), the second step consists in internal behaviour modelling of messages using UML activity diagrams whose state actions are expressed in the $\mathrm{C}++$ Action Language (AL) included in the Rhapsody environment. The AL is a subset of $\mathrm{C}++$ that uses a $\mathrm{C}++$ compiler to enable the model simulation. This language provides message passing, data checking, actions on transitions, and model execution. It supports majority of $\mathrm{C}++$ operators, iflelse, for, while, do/while, return instructions, primitive types, array of primitives, objects, invoking block operations, generating events, generating port events, testing port for an event, etc. Using the Rhapsody environment, we can perform functional simulation before code generation. This step is very important to validate the SystemC code functionality against UML functional models.

6. IMPLEMENTATION AND CASE STUDY We have used the Rhapsody environment for UML modelling and SystemC code generation. In order to automate the SystemC code generation from UML models, we have used the VB API which is integrated in the Rhapsody environment. With VB, we can easily parse UML graphical models then collect the necessary information to create SystemC files. We have developed a VB macro for SystemC code generation and integrated it as a tool box in the Rhapsody environment. As a case study, we have chosen the SDP (Simplex Data Protocol) [6] application whose UML main sequence diagram is illustrated in 
figure 6. Figure 7 gives us an overview of generated SystemC files for the receiver object.

\section{CONCLUSION AND PERSPECTIVES}

In this paper, we present our approach for automatic SystemC code generation from UML models at early stages of SOC design. Our proposed flow consists mainly of two steps: generation of SystemC codes from UML hierarchic sequence diagrams then from UML activity diagrams. Actions of activity diagrams are expressed in the $\mathrm{C}++$ Action Language (AL) which is included in the Rhapsody environment. From AL, a full SystemC code is generated for both simulation and synthesis. SystemC code is generated as text files automatically and this is due to the VB API included in the Rhapsody environment. As a perspective, we plan to investigate the MDA approach for SystemC code generation from Sequence diagrams and consider asynchronous events and temporal constraints.

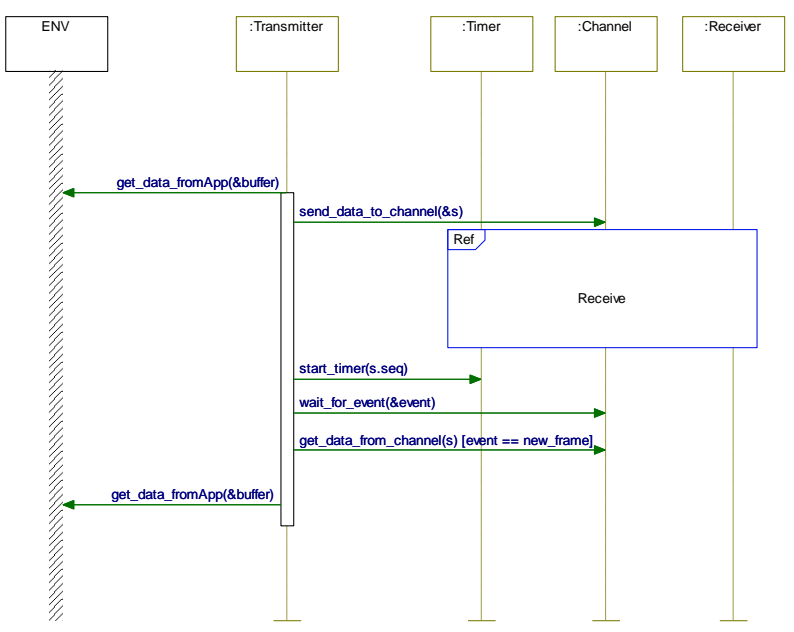

Figure 6. UML sequence diagrams for SDP

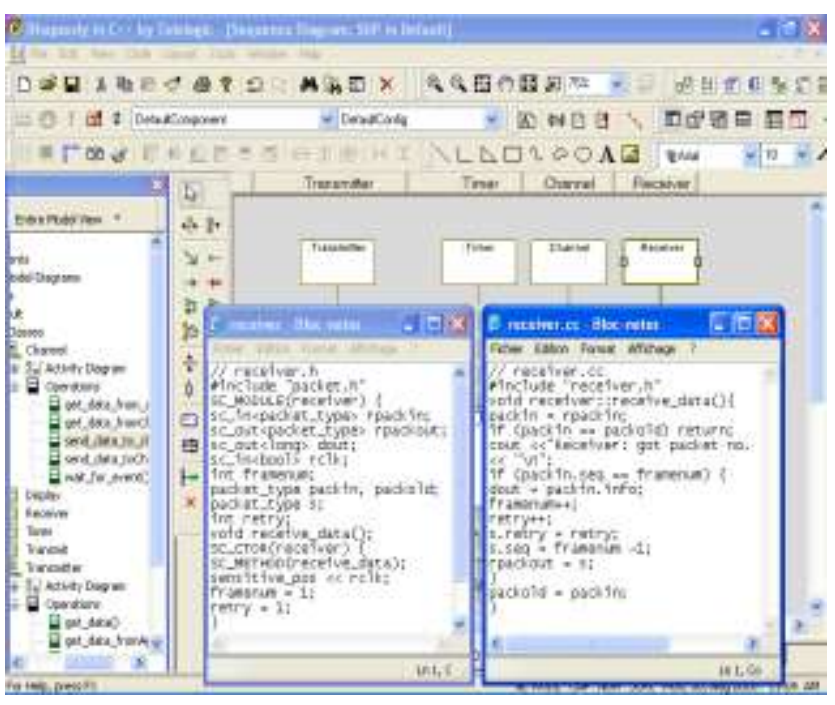

Figure 7. SystemC code generation from Rhapsody UML models

\section{REFERENCE}

[1] Jerraya, A.A. and Wolf, W. 2005. Multiprocessor systems on chip. Morgan Kaufmann publishers.

[2] Booch, G., Rumbaugh, J. and Jacobson I. 1999. Unified Modeling Language User Guide (Addison-Wesley).

[3] UML2.0 Superstructure Specification. 2003, http://www.omg.org

[4] SystemC, IEEE Standard SystemC ${ }^{\circledR}$ language Reference Manual. 2005, www.systemc.org

[5] SystemC, Functional specification for SystemC 2.0. 2002, www.systemc.org

[6] SystemC, Version 2.0 User's guide, 2002, www.systemc.org

[7] Rhapsody UML modeler, www.telelogic.com/products/rhapsody, from Telelogic, an IBM company.

[8] Pauwels, M., et al. A design methodology for the development of a complex system-on-chip using UML and executable system models. 2004. In System Specification \& Design Languages. Springer US.

[9] Nguyen, K. D. Sun, Z., Thiagarajan, P. and Wong W.-F. 2004. Model-driven SOC design via executable UML to SystemC. In IEEE RTSS'04.

[10] Tan, W., et al. Synthesizable SystemC code from UML models. 2004. In UML for Soc Design, DAC 2004 Workshop.

[11] Riccobene, E., Scandura, P., Rosti, A. and Bocchino, S. 2005. A SOC Design Methodology Involving a UML2.0 Profile for SystemC. Proceedings of the Design, Automation and Test in Europe Conference end Exhibition (DATE'05).

[12] Vidal, J., De Lamotte, F., Gogniat, G., Soulard, P. and Diguet, JP. 2009. A codesign approach for embedded system modeling and code generation with UML and MARTE. In DATE09.

[13] Mischkalla, F., He, Da., and Mueller, W. Closing the Gap between UML-based Modeling, Simulation and Synthesis of Combined HW/SW Systems. 2010. In DATE10.

[14] Boutekkouk, F., Benmohammed, M., Bilavarn, S. and Auguin, M. 2009. UML2.0 profiles for Embedded Systems and Systems On a Chip (SOCs). In JOT (Journal of Object Technology).

[15] Coyle, F.P, Thornton, M.A. 2005. From UML to HDL: a Model Driven Architectural Approach to HardwareSoftware Co-Design. Proceedings of Information Systems: New Generations Conference (ISNG), p. 88-93.

[16] Systems Modeling Language (SysML) Specification. 2006. OMG document: ad/2006-03-08-01, version 1. Draft. 